\title{
e-Phaïstos
}

e-Phaïstos

Revue d'histoire des techniques / Journal of the history

of technology

V-1 2016 | 2018

Ville et technique

\section{L'énergie électrique au Sénégal de 1887 à 1985}

Transfert de technologie, appropriation et enjeu politique d'un patrimoine industriel naissant

Electrical Energy in Senegal,1887-1985. Technology Transfer, Appropriation and Political Stake of a Rising Industrial Heritage

\section{Salif Diedhiou}

\section{(2) OpenEdition}

\section{Journals}

Édition électronique

URL : http://journals.openedition.org/ephaistos/1209

DOI : 10.4000/ephaistos. 1209

ISSN : 2552-0741

Éditeur

IHMC - Institut d'histoire moderne et contemporaine (UMR 8066)

Référence électronique

Salif Diedhiou, «L'énergie électrique au Sénégal de 1887 à 1985 », e-Phaïstos [En ligne], V-1 2016 |

2018, mis en ligne le 21 janvier 2018, consulté le 09 novembre 2019. URL : http://

journals.openedition.org/ephaistos/1209

Ce document a été généré automatiquement le 9 novembre 2019

Tous droits réservés 


\section{L'énergie électrique au Sénégal de 1887 à 1985}

Transfert de technologie, appropriation et enjeu politique d'un patrimoine industriel naissant

Electrical Energy in Senegal,1887-1985. Technology Transfer, Appropriation and Political Stake of a Rising Industrial Heritage

Salif Diedhiou

Thèse : références bibliographiques

Salif Diedhiou, L'énergie électrique au Sénégal de 1887 à 1985. Transfert de technologie, appropriation et enjeu politique d'un patrimoine industriel naissant, Thèse de doctorat de l'École Pratique des Hautes Études, mention Histoire, textes et Documents, École doctorale 472, EA 4116 « Savoirs et Pratiques du Moyen Âge au XIXe siècle » (SAPRAT), Paris Sciences et Lettres PSL Research University, soutenue le 25 juin 2016, un volume (434 pages)

\section{Directeur de thèse}

Jean-François Belhoste, Directeur d'Études, École Pratique des Hautes Études

Jury

Mbaye Thiam, Professeur, École des Bibliothécaires Archivistes et Documentalistes (EBAD) de l'Université Cheik Anta Diop de Dakar, (président)

Anne-Françoise Garçon, Professeur des Universités, Université Paris 1 PanthéonSorbonne (rapporteur)

Alain Beltran, Directeur de recherche, Centre national de la recherche scientifique (rapporteur)

Paul Smith, Chargé de Mission, Ministère de la Culture et de la Communication, (examinateur) 
1 Monsieur le Président, messieurs les membres du jury. Je vous remercie de l'intérêt que vous avez portez à ce travail sur L'énergie électrique au Sénégal de 1887 à 1985 et de votre présence aujourd'hui afin de le discuter. Je remercie également tous ceux qui m'ont fait l'amitié de venir assister à la soutenance de la thèse.

2 Des responsabilités au ministère de la culture du Sénégal m’ont conduit à me poser des questions sur la place du patrimoine industriel dans la politique patrimoniale du Sénégal. La thèse que j'ai l'honneur de présenter, n'est pas le fruit du hasard. Elle constitue l'aboutissement d'un parcours professionnel et universitaire, après des études académiques en histoire des techniques et dans les métiers du patrimoine, en France, en Espagne, au Portugal et en Italie, récemment publiées sur le chemin de fer et la distribution de l'eau dans les grandes villes coloniales du Sénégal. Opter pour l'électrification, thématique devenue d'actualité, avec la Cop 21 et ou le projet Energies pour l'Afrique porté par Jean-Louis Borloo, permet, en effet, de faciliter l'acceptation d'un patrimoine industriel, souvent associé au passé colonial.

3 Pourquoi basculer d'un sujet de Master 2 Erasmus Mundus, TPTI Techniques, Patrimoines, Territoires de l'Industrie de l'Université Paris 1 (2011) sur le chemin de fer au Sénégal vers cette thématique toujours prégnante de l'électrification du pays dans la longue durée? Les émeutes de l'électricité de 2010 au Sénégal constituent bien l'évènement historique déclencheur (plus qu'un fait divers) en me conduisant à me poser la question de la raison de ces insurrections à Dakar et dans d'autres villes du pays, au bout d'un siècle d'électrification. Le nœud de la thèse se situe bien dans cette problématique socioéconomique. Pourtant, il y a un lien entre l'étude des deux secteurs d'activité, le chemin de fer (en 2011) et l'électrification (en 2016) : l'étude sur le chemin de fer m'a permis de comprendre la différence de méthode de transfert et d'appropriation technique entre les deux secteurs.

4 Comment s'est donc déroulé le processus d'électrification du Sénégal ? En une première électrification, inscrite dans la phase de la transplantation des techniques qui fait l'objet d'une première partie. L'unification du réseau et des unités de production apparait à propos de l'électrification qui est étudié en seconde partie. En élargissant la focale autour des premières usines et de la "sénégalisation " des postes techniques, apparaît alors le transfert de technologie dans toute son amplitude en un second temps. Enfin dans un dernier volet, et au terme de cette électrification, la question se pose de ce que sont désormais ces usines de production: ont-elles accédé au statut de patrimoine industriel?

5 J'ai choisi d'inscrire mon travail dans une démarche thématique, avec les incontournables supports chronologiques que sont le passé sous l'exploitation française (de la $1^{\text {ère }}$ moitié du XIX ${ }^{e}$ siècle jusqu'à l'indépendance du pays en 1960), puis l'impact de la décolonisation avec une première phase de transition et de négociations avec la Compagnie des Eaux et Electricité Ouest Africain (E.E.O.A), concessionnaire en titre, et une seconde phase de prise en main par les autorités sénégalaises du secteur de l'énergie, avec le soutien d'EDF. J'ai ainsi souhaité mettre en avant le jeu d'acteurs depuis le début du processus de colonisation jusqu'au transfert de technologie et mettre en valeur un espace de grande mutation énergétique où l'innovation et le passé se côtoient sans réellement dialoguer, sauf à les y inviter par des actions de mise en valeur patrimoniale. 
6 Trois démarches ont été adoptées : Tout d'abord, j'ai constitué une base biographique des principaux acteurs de l'électrification, les ingénieurs et les administrateurs qui ont joué un rôle central dans l'électrification en utilisant les instruments documentaires et les fonds des Archives nationales françaises à Paris et ceux des Archives d'outre-mer à Aix-en-Provence. Si cette récolte s'est avérée assez maigre, elle a cependant permis de dresser le parcours de chacun des deux premiers concessionnaires. Ensuite j'ai établi d'une part, un corpus de sources imprimées (publications spécialisées, revues, articles etc.), et d'autre part un corpus d'archives d'institutions et d'entreprises. Les fonds des Archives nationales du Sénégal, celles d'EDF et du Conservatoire national des Arts et Métiers ont été mobilisés, de même que ceux des bibliothèques parisiennes (BNF, Cnam, Cité des sciences et de l'Industrie ou Centre de documentation de la Fondation EDF). Enfin, j'ai adopté la méthode de l'archéologie industrielle et de l'enquête, en effectuant une visite in situ de l'usine Sunéor de Ziguinchor (Casamance). En immersion, j'ai découvert les traces matérielles et le ressenti humain de ce passé industriel, dans une usine toujours en activité. Ces investigations ont été fructueuses, quoiqu'elles m'aient valu quelques déboires, pour obtenir les autorisations de pénétrer dans l'enceinte de l'usine par exemple ..., mais qui sont inhérents à l'approche de terrain.

7 Les difficultés majeures rencontrées dans la recherche proprement dite ont été l'accessibilité aux archives d'entreprises de la Société Nationale d'Electricité du Sénégal (SENELEC) et de la Centrale de Sunéor, l'absence d'archives pour les d'entreprises disparues (la Société de Vaubourg de Saint Louis, la Compagnie d'Électricité du Sénégal (CEL) d'André De Traz, la Compagnie Eau Électricité Ouest Africaine (EEOA), leurs archives de gestion n'ayant été versées ni dans les institutions d'archives françaises ni aux Archives nationales du Sénégal. Cet écueil, propre aux systèmes de collecte et de classement d'archives, qui constitue un impondérable de la recherche, m'a obligé à emprunter d'autres voies, et provoqué d'évidence un déséquilibre entre les parties, certains chapitres étant largement plus documentés que d'autres. À titre d'exemple la législation et les contrats de la fin du XIX sont quasiment dépourvus de sources primaires, notamment pour la concession de Saint-Louis entre Vaubourg et la commune de Saint Louis. De même, nulle source ne paraissait mobilisable pour renseigner les promoteurs et le personnel technique de la Compagnie EEOA dont a hérité l'État du Sénégal, ce qui aurait été apprécié pour compléter l'approche humaine. Ce travail trouve ici ses limites: lier la situation présente au passé semble un exercice complexe, que vient brouiller un peu plus dans la phase introductive le mythe de la Fée Electricité et/ou l'image de l'Afrique en France et en Occident telle que l'une et l'autre se sont construites dans les expositions coloniales et universelles, que je n'évoquerais plus ensuite mais dont les symboliques culturelles, sociales et techniques sont indéniables.

8 L'écueil matériel n'est pas à négliger dans la réalisation de la thèse. Il nous aurait fallu un peu plus de temps lors de l'étude archéologique pour aller au-delà de l'analyse des infrastructures et aborder l'analyse des imaginaires des ingénieurs, des cadres et des ouvriers, ce qui aurait requis également plus de moyens. Le financement de la thèse s'est avéré une contrainte majeure. En effet, bien que soutenu - et j'en remercie vivement les institutions - par des bourses d'études de la fondation EDF et de la Région Île-de-France, le recours à d'autres expédients pour parvenir au terme de la thèse s'est imposé (emplois de nuit difficilement compatibles avec une recherche documentaire, archivistique et scientifique sereine, enchaînée par le parcours doctoral en ses diverses 
modalités). Être ici aujourd'hui apporte la preuve que j'ai surmonté en partie ces écueil et déboires, me permettant de dégager certaines conclusions.

La thèse retrace d'abord le contexte et le processus de l'introduction des techniques occidentales dans la colonie du Sénégal et de l'Afrique Occidentale française (AOF) plus généralement, avec les phases de son développement, les vecteurs de la technique et les secteurs qui ont permis son développement et son appropriation. Alors que pour le secteur ferroviaire, certaines aptitudes de métiers traditionnels, liées au travail du bois et $\mathrm{du}$ fer, permettaient l'adaptation aux techniques occidentales, l'équivalent de ces métiers n'existant pas dans les métiers de l'électricité, le transfert de technologie dans ce secteur prit en conséquence une toute autre dimension. Le processus s'avéra beaucoup plus complexe, en particulier la gestion des techniques électriques. À l'indépendance, la politique de transfert de technologie de l'énergie électrique a été définie conjointement par l'État du Sénégal et Électricité de France (EDF). Le transfert s'est alors centré sur les personnels locaux grâce à une formation ad hoc. Au Sénégal l'équipement appartenait à l'ancien concessionnaire, la Compagnie EEOA. Pour nationaliser l'outil de production, l'État confia la formation du personnel technique à EDF : certes l'équipement existait mais la formation des techniciens locaux s'imposait. Je conclue que ce processus s'est en réalité achevé beaucoup plus tardivement, puisque d'autres technologies ont été introduites et conduites par EDF tel que le dispatching qui a nécessité d'autres dispositions.

10 Vient ensuite la question du patrimoine industriel et plus particulièrement de la valorisation des infrastructures, symbole de l'histoire industrielle du Sénégal. J'observe que la démarche entreprise auprès des responsables de l'usine Sunéor de Ziguinchor (Casamance), cas de notre étude, a été rendu possible par une véritable collaboration avec les cadres, ce qui a facilité l'enquête et permis de convoquer la mémoire des anciens ouvriers et agents, appelés au secours d'une démarche d'archéologie "en action ». Je suis intervenu, en effet, dans une usine encore en activité. L'intérêt s'est situé également dans l'étude de l'hybridation si prolixe au Sénégal. Ainsi, un improbable soutien de l'énergie tirée de l'arachide permet de pallier le défaut de ressource, preuve d'une adaptation et d'un imaginaire inné de la technique. À partir de l'intérêt suscité par cette recherche, les responsables de l'usine envisagent d'ailleurs un programme structuré de visites d'entreprises tenant compte de ce volet du patrimoine industriel, désormais conçu comme un atout pour l'entreprise.

11 Conclusion: Malgré les déboires et les écueils, ces années de recherche ont été pour moi une période d'épanouissement intellectuel. Faire l'histoire de l'électricité sans en avoir les notions techniques peut sembler une gageure au point que certains me demandaient en bout de course si j'étais ingénieur du domaine. Il m'a fallu découvrir et intégrer ces notions de base pour comprendre le langage des objets, leur interrelation, leur système technique. J'espère avoir contribué à une meilleure compréhension de l'histoire de l'électrification du Sénégal et aidé à percevoir les enjeux politiques du patrimoine industriel, fortement lié à l'histoire économique et sociale de l'Afrique telle qu'elle est structurée de nos jours. Mais j'espère également avoir donné une vision plus volontaire de nos approches, consciente d'un passé avec lequel il faut composer et avancer, cela dans le domaine de l'archéologie industrielle comme dans d'autres. C'est en tout cas dans cette direction que j'espère pouvoir mener des recherches futures, puisque mes sujets sont au cœur des débats actuels sur notre continent. 

étrangère à l'exacerbation des émeutes de l'électricité en 2010. Suite aux délestages chroniques du courant électrique, l'État du Sénégal a décidé en 2010 de signer un accord avec EDF afin de réaliser le diagnostic du réseau électrique du pays. Les ingénieurs d'EDF, arrivés en 2010, ont trouvé sur place les 450 ingénieurs maison de la SENELEC, qui d'évidence ne leur ont pas facilité la tâche, puisqu'ils étaient dans une logique de contestation. Ne maîtrisant pas l'outil, les ingénieurs d'EDF n'ont pas obtenu les résultats escomptés, cela malgré le coût élevé du contrat. La rumeur a vite gonflé, portée par les syndicats. La presse s'en est saisi et des superlatifs les plus incroyables ont fusé : mépris colonial, recolonisation de l'électricité, etc... qu'il convient ici de nuancer. On aura donc manqué du recul historique nécessaire pour éviter cette situation et les dérives qui s'en ont suivies, aussi bien de la part de l'État que des principaux acteurs en présence, dans les structures de production mais également dans l'opinion publique. L'appropriation par les décideurs et les médias de travaux historiques comme cette thèse sur L'énergie électrique au Sénégal de 1887 à 1985 aurait-elle permis d'éviter pareille situation ou d'en atténuer les retombées? Je ne peux que l'espérer, comme j'espère que ce travail sera reconnu et apprécié sur place, mieux qu'un vœu pieux vite oublié, et qu'il sera perçu comme un atout mis à disposition des tous et chacun.

Je vous remercie vivement de votre attention et me réjouis d'échanger avec vous, Monsieur le président et Mesdames et Messieurs les membres du jury, à propos de ce travail.

\section{RÉSUMÉS}

L'électrification du Sénégal est intervenue assez tôt, en 1889, à Saint-Louis, au moment où les grandes villes européennes n'avaient pas fini de découvrir toutes les possibilités qu'offrait cette grande innovation énergétique. L'industrie de l'électricité est ainsi restée aux mains d'entrepreneurs métropolitains jusqu'à la fin des années 1970. Durant toute cette période, le personnel technique, en charge de son fonctionnement a été essentiellement européen. C'est avec le processus de nationalisation du secteur, amorcé en fin 1972, qu'on a posé pour la première fois l'option de la «sénégalisation » des postes techniques, et ainsi du processus de transfert de technologie de l'énergie électrique vers le personnel local. Cette mission a été conduite par Électricité de France, en accord avec l'État du Sénégal. Il a néanmoins fallu presqu'un siècle, de 1889 à 1985, pour que le Sénégal s'approprie la technologie. Cette thèse, analyse à la fois l'introduction des techniques depuis la colonisation mais également la trajectoire de l'électrification du Sénégal et les outils du transfert de la technologie liée à l'énergie électrique. Elle évoque en outre la politique patrimoniale et l'enjeu du patrimoine industriel dans un pays non industriel comme le Sénégal.

The electrification of Senegal had already occurred in 1889, in Saint-Louis, at a time when the European cities had yet to discover all the possibilities that this great technological innovation comprised. The industry of electricity thus remained in the hands of metropolitan entrepreneurs until the end of the 1970s. During this period, the technical personnel in charge of its operating 
were essentially European. It is with the nationalization process launched in the seventies that, for the first time, the term "senegalisation" is coined; and, with it, the process of technology transfers to the local personnel. Electricity de France, in agreement with the State of Senegal, has conducted this task. It nevertheless took close to a century, from 1889 to 1985, for Senegal to fully seize the technology. This thesis analyzes the introduction of techniques since the colonization and both the path of electrification of Senegal, alongside the tools necessary to the transfer of the technology of electrical energy. It further mentions the patrimonial policy, and the stakes of the industrial heritage in a non-industrial country such as Senegal.

\section{INDEX}

Mots-clés : histoire des techniques, électricité, électrification, ville coloniale, patrimoine colonial, transfert de technologie

Keywords : history of technology, electricity, electrification, colonial town, colonial heritage, industrial heritage

Thèmes : Positions de thèse 